\title{
Characterizing the Role of Biologically Relevant Fluid Dynamics on Silver Nanoparticle Dependent Oxidative Stress in Adherent and Suspension In Vitro Models
}

\author{
Katherine E. Burns, Robert F. Uhrig, Maggie E. Jewett, Madison F. Bourbon and Kristen A. Krupa *D
}

check for

updates

Citation: Burns, K.E.; Uhrig, R.F.; Jewett, M.E.; Bourbon, M.F.; Krupa, K.A. Characterizing the Role of Biologically Relevant Fluid Dynamics on Silver Nanoparticle Dependent Oxidative Stress in Adherent and Suspension In Vitro Models. Antioxidants 2021, 10, 832. https:// doi.org/10.3390/antiox10060832

Academic Editor: Tim Hofer

Received: 5 May 2021

Accepted: 21 May 2021

Published: 23 May 2021

Publisher's Note: MDPI stays neutral with regard to jurisdictional claims in published maps and institutional affiliations.

Copyright: (c) 2021 by the authors. Licensee MDPI, Basel, Switzerland. This article is an open access article distributed under the terms and conditions of the Creative Commons Attribution (CC BY) license (https:/ / creativecommons.org/licenses/by/ $4.0 /)$.
Department of Chemical and Materials Engineering, University of Dayton, Dayton, OH 45469-0256, USA; burnsk7@udayton.edu (K.E.B.); uhrigr1@udayton.edu (R.F.U.); jewettm3@udayton.edu (M.E.J.); mbourbon1@udayton.edu (M.F.B.)

* Correspondence: kkrupa1@udayton.edu; Tel.: +1-937-229-2627

Abstract: Silver nanoparticles (AgNPs) are being employed in numerous consumer goods and applications; however, they are renowned for inducing negative cellular consequences including toxicity, oxidative stress, and an inflammatory response. Nanotoxicological outcomes are dependent on numerous factors, including physicochemical, biological, and environmental influences. Currently, NP safety evaluations are carried out in both cell-based in vitro and animal in vivo models, with poor correlation between these mechanisms. These discrepancies highlight the need for enhanced exposure environments, which retain the advantages of in vitro models but incorporate critical in vivo influences, such as fluid dynamics. This study characterized the effects of dynamic flow on AgNP behavior, cellular interactions, and oxidative stress within both adherent alveolar (A549) and suspension monocyte (U937) models. This study determined that the presence of physiologically relevant flow resulted in substantial modifications to AgNP cellular interactions and subsequent oxidative stress, as assessed via reactive oxygen species (ROS), glutathione levels, p53, NFkB, and secretion of pro-inflammatory cytokines. Within the adherent model, dynamic flow reduced AgNP deposition and oxidative stress markers by roughly $20 \%$. However, due to increased frequency of contact, the suspension U937 cells were associated with higher NP interactions and intracellular stress under fluid flow exposure conditions. For example, the increased AgNP association resulted in a 50\% increase in intracellular ROS and p53 levels. This work highlights the potential of modified in vitro systems to improve analysis of AgNP dosimetry and safety evaluations, including oxidative stress assessments.

Keywords: silver nanoparticle; reactive oxygen species; cytotoxicity; dynamic flow; p53; NFkB; cytokine secretion

\section{Introduction}

Due to their physicochemical properties, nanoparticles (NPs) possess unique behavioral traits, such as augmented reactivity, increased transport potential, and distinctive optical signatures. The utilization of these properties and behavioral characteristics has resulted in the incorporation of NPs into hundreds of consumer products and applications [1]. To date, silver NPs (AgNPs) are the most predominantly employed, with applications spanning the medical, electrical, industrial, consumer goods, and health care sectors [2,3]. Owing to their robust antibiotic and antifungal activities, AgNPs have been integrated into bandages, biomedical devices, food storage apparatuses, textiles, cosmetics, and material surface coatings [4,5]. Additionally, their plasmonic capabilities have made AgNPs attractive for biomedical applications including bioimaging, optical sensor development, drug delivery vehicles, and as a mechanism to harvest photonic energy [5,6].

As NP prevalence increases, a corresponding rise in human contact also occurs, whether intentionally via therapeutics or via unintentional introduction. Furthermore, 
AgNPs have been linked to potential health concerns, with exposure resulting in significant cytotoxicity [7]. In addition to cellular death, AgNP exposure has been shown to induce numerous bioeffects including augmented oxidative stress, activation of the inflammatory and immune responses, alterations to signal transduction pathways, and genetic modifications [8-11]. For example, a study by Comfort et al. demonstrated that, even at dosages in the $\mathrm{pg} / \mathrm{mL}$ range, AgNPs were able to disrupt normal cell homeostasis by augmenting cellular stress, activating Akt and Erk signal transduction pathways, stimulating pro-inflammatory cytokine secretion, and regulating gene expression [9]. In a recent study by Khan et al., $55 \mathrm{~nm}$ AgNPs were shown to penetrate the blood brain barrier and induce oxidative stress pathways and production of several proteins involved in neurodiseases [12]. In addition to cellular death, AgNP exposure has been linked to disruption of the human microbiota, autoimmune diseases, and environmental health concerns, highlighting the far-reaching implications of AgNP exposure [13,14] Given the potential for serious health and environmental complications, it is necessary that the cellular response to NP exposure be fully characterized and understood. However, cataloging the safety of NPs is a major challenge as bioresponses are dependent upon a unique combination of physicochemical properties, such as primary size, surface chemistry, core composition, and morphology $[15,16]$. This means that each AgNP set has the potential to induce a distinctive cytotoxic profile within a biological environment.

In addition to NP physicochemical properties, the biological exposure environment also plays a significant role in how cells recognize and interact with NPs. For example, the type of cell, available surface receptors, and surrounding environmental factors all contribute differently to the NP protein corona and nano-cellular interface, and the subsequent cytotoxic outcomes $[17,18]$. In a recent study, it was determined that two different hepatic cell models uniquely interacted and displayed differential cytotoxicity following exposure to identical gold nanoparticles [17]. Currently, NP safety assessments are carried out in either cell-based in vitro or animal-based in vivo models. While in vitro systems possess experimental flexibility, are cost-effective, and have high throughput capabilities, they significantly lack physiological relevance [19]. The discrepancy between these models has resulted in poor correlation of nanotoxicological results, with NPs inducing more diverse and greater cellular responses in vitro versus in vivo counterparts [20-24].

One approach to overcome the poor correlation between cell and animal systems is to develop enhanced biological models, which retain the in vitro advantages but incorporate key in vivo influences to produce a more realistic and relevant NP exposure scenario. One example is the inclusion of dynamic fluid movement, which can mimic the biological transport mechanisms of cardiovascular flow, and thereby include a more accurate prediction of NP transport. Initial dynamic studies identified that lateral fluid flow altered the balance between NP diffusion and sedimentation, thereby modifying dosimetry and resultant bioresponses $[25,26]$. A study by Fede et al. demonstrated that under static conditions gold nanoparticles had a higher rate of sedimentation and cytotoxicity than when exposure occurred within a dynamic flow environment [26]. The incorporation of fluid dynamics is critical in biological models that experience high rates of fluid motion, such as the blood brain barrier and vascular systems $[27,28]$. Additionally, exposure within a dynamic environment has been found to alter the protein corona, the recognized biological identity of NPs $[29,30]$. While several studies have examined the role of fluid dynamics on adherent cell models, to date, no work exists on how fluid dynamics influences the NP cellular association, stress, and toxicity within a suspension cell model.

The goal of this study was to elucidate and characterize the impact of dynamic flow on AgNP behavior, deposition, and biological response in both an adherent (A549) and a suspension (U937) human cell model. Lung cells, A549, were specifically chosen as inhalation is a primary form of NP exposure and A549s have been widely utilized in nanotoxicological evaluations [31]. The monocytic U937 cell line was of particular interest as monocytes are primary responders to foreign material in the body and trigger systemic oxidative and inflammatory responses [32]. Fluid flow within the cellular systems was 
generated using a peristaltic pump, which produced a tube-side linear velocity of 0.2 $\mathrm{cm} / \mathrm{s}$, equivalent to known capillary rates [33]. This study demonstrated that the presence of dynamic flow impacted AgNP deposition and the ensuing cellular responses for the experimental $50 \mathrm{~nm}$ AgNPs. In particular, several intracellular oxidative stress pathways were activated within both the A549 and U937 models. Interestingly, the adherent and suspension cells responded inversely to dynamic flow, with the A549 cells correlating to lower AgNP interactions and stress with fluid dynamics. It is hypothesized that, under the influence of fluid dynamics, the suspension U937 cells experienced augmented interactions with flowing AgNPs, thereby inducing more significant oxidative stress outcomes.

\section{Materials and Methods}

\subsection{AgNP Characterization}

The experimental $50 \mathrm{~nm}$ polyvinylpyrrolidone (PVP)-coated AgNPs were purchased from nanoComposix in concentrated solution form. nanoComposix high quality control guarantees that their products are endotoxin free. To minimize AgNP modifications over the duration of experimentation, the particles were stored at $4{ }^{\circ} \mathrm{C}$ in the dark. Transmission electron microscopy (TEM) analysis was performed on a Hitachi H-7600 to verify primary particle size and morphology.

For the remaining AgNP characterization assessments, the particles were diluted to a concentration of $25 \mu \mathrm{g} / \mathrm{mL}$ in the denoted fluid. The unique spectral signatures of these particles were visualized via ultraviolet visible (UV-Vis) spectroscopy on a Synergy 4 BioTek microplate reader. Degree of agglomeration was measured through dynamic light scattering (DLS) on an Anton Paar Litesizer 500. The Anton Paar Litesizer was also used to run zeta potential analysis in order to determine particle surface charge.

\subsection{Mammalian Cell Culture}

Both human cell lines utilized in this study, the lung epithelial A549 and the monocytic U937, were purchased from American Type Culture Collection. The cells were maintained in RPMI 1640 media supplemented with 10\% fetal bovine serum (FBS) and 1\% antibiotics. The A549 cells, which are adherent in nature, were cultured on tissue-culture-treated petri dishes. The U937s were grown in suspension in T-75 tissue culture flasks. Both cells lines were sub-cultured every 3 to 4 days, as necessary.

\subsection{Establishment of Dynamic Environment}

A multi-channel peristaltic pump (Ismatec-Wertheim Germany, model \#ISM939D) was used to establish dynamic flow, as previously demonstrated [25]. Both inlet and outlet ends of the tubing, with a $1 / 16$ inch inner diameter, were secured into individual wells of a 24-well plate to generate unilateral flow throughout the cellular systems. Prior to experimentation, protein-rich media were run through the system to coat the tubes, thereby minimizing unintentional AgNP binding. For A549 experimentation, the pump was primed with either untreated or AgNP-dosed media to ensure that media levels were equivalent for both static and dynamic conditions. As U937 cells are suspension cells by nature, the pump was also primed prior to experimentation, but additional cells were included in order to maintain a constant cellular density with respect to media volume. To ensure no alterations in temperature, the pump and tubing were stored within the incubator. During experimentation, the pump operated at a rate that produced a tube-side linear velocity of $0.2 \mathrm{~cm} / \mathrm{s}$, which was specifically chosen to match physiological rates within capillaries [33].

\subsection{Nano-Cellular Association}

To determine AgNP interaction with the biological systems, cells were seeded into 24-well plates $\left(2 \times 10^{5}\right.$ A549 cells/well or $1.5 \times 10^{5}$ cells $/ \mathrm{mL}$ for U937) and incubated overnight. The denoted cells were exposed to $15 \mu \mathrm{g} / \mathrm{mL}$ of the $50 \mathrm{~nm}$ AgNPs, under static or dynamic conditions. This AgNP concentration was selected as it was large enough 
to provide a significant detection signal but did not induce a strong cytotoxic response. Following 24 h exposure, the AgNP-containing media was collected; the U937 cells were removed via low-speed centrifugation, which removed the cells but not the suspended AgNPs. The supernatant then underwent UV-VIS analysis to determine the final NP concentration, using previously generated concentration curves [34]. A separate calibration curve was generated for static and dynamic conditions to account for any non-specific binding that may have occurred under dynamic conditions. The difference between this final concentration and the administered $15 \mu \mathrm{g} / \mathrm{mL}$ dosage was the AgNP amount associated with the cells.

\subsection{Cellular Viability}

Cell viability was determined using the CytoTox 96 Non-Radioactive Cytotoxicity Assay (Promega), which measures the production of lactate dehydrogenase (LDH). A549 cells were plated into 24-well plates at a concentration of $2 \times 10^{5}$ cells per well. U937s were seeded into 24 -well plates at a density of $1.5 \times 10^{5}$ cells $/ \mathrm{mL}$, as they were in suspension. The following day, the cells underwent exposure to the $50 \mathrm{~nm}$ AgNPs at 0, 5, or $25 \mu \mathrm{g} / \mathrm{mL}$, under either static or dynamic conditions. After $24 \mathrm{~h}$, the media were removed and centrifuged to remove AgNPs and suspension cells. The supernatant then underwent LDH evaluation in accordance with the manufacturer's instructions. A positive toxicity control, in which all the cells were lysed, was used to normalize data and determine degree of cytotoxicity.

\subsection{Intracellular ROS Production}

Intracellular stress was assessed by measuring reactive oxygen species (ROS) levels following AgNP exposure under the denoted conditions. A549 and U937 cells were seeded in 24-well plates at $2 \times 10^{5}$ cells per well or $1.5 \times 10^{5}$ cells $/ \mathrm{mL}$, respectively, and returned to the incubator until the following day. Next, the cells were washed and incubated with the DCFH-DA probe (Thermo Fisher Scientific) for $30 \mathrm{~min}$, washed again, then dosed with the AgNPs at 0, 5, or $25 \mu \mathrm{g} / \mathrm{mL}$ within either a static or dynamic environment. After $24 \mathrm{~h}$ incubation, the ROS levels were measured via fluorescent analysis using a Synergy 4 BioTek microplate reader. Untreated cells under static conditions served as the negative control for normalization and hydrogen peroxide-dosed cells served as a positive control.

\subsection{Glutathione Levels}

The ratio of reduced glutathione (GSH) to oxidized glutathione (GSSG) is a metric to quantify intracellular oxidative stress [35]. The experimental cells were seeded and exposed to 0, 5, or $25 \mu \mathrm{g} / \mathrm{mL}$ AgNPs as described above for ROS. After $24 \mathrm{~h}$, the cells were then washed, collected, and lysed in a non-denaturing lysis buffer. Intracellular GSH and GSSG levels were quantified using the GSH/GSSG Ratio Detection Assay Kit from Abcam, using a Synergy 4 BioTek microplate reader. GSH/GSSG ratios were calculated and normalized against an untreated, static control for each cellular system. Hydrogen peroxide exposure served as a positive control for known stress activation.

\subsection{Activation of Intracellular Targets}

A549 and U937 cells were seeded in a 24-well plate at $2 \times 10^{5}$ cells per well or $1.5 \times 10^{5}$ cells $/ \mathrm{mL}$, respectively. The following day the cultures were dosed with 0,5 , or $25 \mu \mathrm{g} / \mathrm{mL}$ AgNPs, under either static or dynamic conditions. After $24 \mathrm{~h}$, the cells were washed, collected, and lysed in a non-denaturing lysis buffer. Activation of intracellular p53 and NFkB were determined by quantifying phosphorylated levels of these targets. In both cases, phosphorylated levels were normalized by the total p53 and NFKB amounts, quantified in parallel. Phospho and total levels of p53 and NFKB were assessed using protein-specific PathScan ELISA kits from Cell Signaling Technology, in accordance with the manufacturer's instructions. Experimental activation levels were normalized against 
untreated, static controls. Hydrogen peroxide-exposed cells served as a positive control as they are known to induce numerous intracellular stress pathways.

\subsection{Inflammatory Cytokine Secretion}

The inflammatory response to AgNP exposure was determined by quantifying the production of the cytokines interleukin-1 $\beta$ (IL-1 $\beta$ ) and tumor necrosis factor- $\alpha$ (TNF- $\alpha$ ). While a panel of cytokines are secreted within an inflammatory response, IL-1 $\beta$ and TNF- $\alpha$ were selected as representative protein markers for this assessment. A549 and U937 cells were seeded in 24 -well plates at $2 \times 10^{5}$ cells per well or $1.5 \times 10^{5}$ cells $/ \mathrm{mL}$, respectively, incubated for $24 \mathrm{~h}$, then exposed to AgNPs under the stated conditions. Hydrogen peroxide exposure served as a positive control as it is known to induce numerous intracellular stress pathways. After $24 \mathrm{~h}$ exposure, the media were collected, AgNPs/U937s were removed via centrifugation, and the supernatant underwent analysis for extracellular IL- $1 \beta$ and TNF- $\alpha$ levels using protein-specific ELISAs (Thermo Fisher Scientific, Waltham, MA, USA), in accordance with the manufacturer's directions.

\subsection{Data Analysis}

All data are presented as the mean \pm the standard error of the mean. All experimentation was carried out with three independent trials. For cellular association analysis, a one-way ANOVA with Bonferroni post-test was run using GraphPad Prism to determine statistical significance between static and dynamic conditions. For the remaining experimentation, a two-way ANOVA with Bonferroni post-test was run using GraphPad Prism, with * and + indicating significance compared to the untreated control and between static and dynamic conditions, respectively.

\section{Results}

\subsection{AgNP Characterization}

Prior to cellular exposure, the AgNPs underwent numerous characterization assessments to quantify the unique physicochemical properties associated with this NP stock. As small deviations in NP properties have been correlated to differential bioresponses, it was necessary to first characterize the experimental NPs [36]. A representative TEM image of the $50 \mathrm{~nm}$ AgNPs is shown in Figure 1 and demonstrates a uniform, spherical morphology. Using multiple images, the primary particle size was determined to be $52.6 \pm 6.9 \mathrm{~nm}$. The uniformity of the AgNP stock was further verified through spectral analysis, which was comprised of a single, sharp peak (Figure 1B). When dispersed in media, there is a very slight right-shift in the spectral image, which can be attributed to the formation of a protein corona or minimal particle agglomeration.
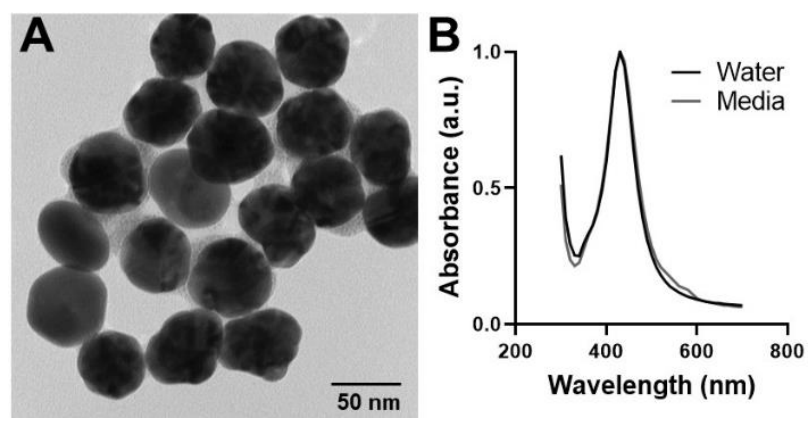

Figure 1. Characterization of the experimental $50 \mathrm{~nm}$ AgNP stock. (A) A representative TEM image of the $50 \mathrm{~nm}$ PVP-coated AgNPs is shown. This image verifies spherical morphology and an even particle size distribution. (B) The spectral profiles for the $50 \mathrm{~nm}$ AgNPs in both water and media demonstrate a sharp, single peak, further verifying particle uniformity. 
In addition to verifying the primary particle size, the agglomerate sizes were determined in both water and media (Table 1). As all NPs will agglomerate when in solution, it is important to assess the extent of agglomeration as it can impact mechanisms of biotransport [37]. The AgNPs displayed minimal agglomeration in water with an increased effective diameter in media, due to the formation of a protein corona [38]. As the NPs had a PVP surface coating, which is known to promote particle stability in solution, the small extent of aggregation was expected [39]. Additionally, the surface charge was assessed using zeta potential measurements. The stock AgNPs displayed a negative surface charge, approximately $-30 \mathrm{mV}$. Following the formation of a protein corona in media the charge shifted to $-10 \mathrm{mV}$, as the outermost NP surface was covered in proteins which innately have a slight negative charge [38].

Table 1. Characterization of the AgNPs under static and dynamic conditions.

\begin{tabular}{ccccc}
\hline Flow Condition & \multicolumn{2}{c}{ Agglomerate Size (NM) } & \multicolumn{2}{c}{ Zeta Potential (MV) } \\
& Water & Media & Water & Media \\
\hline Static & $78.6 \pm 3.0$ & $89.3 \pm 2.2$ & $-30.4 \pm 1.7$ & $-9.7 \pm 0.6$ \\
Dynamic & $76.7 \pm 2.4$ & $90.8 \pm 2.9$ & $-32.0 \pm 2.4$ & $-10.5 \pm 1.0$ \\
\hline
\end{tabular}

A peristaltic pump was used to generate fluid dynamics within the cellular system. Pump utilization afforded the opportunity to assess the impact of fluid dynamics on both AgNP characteristics and the nano-cellular interface. The targeted pump flow rate produced a linear velocity of $0.2 \mathrm{~cm} / \mathrm{s}$ within the tubing, aligning with known capillary values. This means that the velocity across the cells was orders of magnitude less, aligning with the diffusion-based transport observed within tissue. Next, the AgNPs, suspended in either water or media, were added into an acellular dynamic system, circulated for several hours, and characterized in order to determine if the shear stress impacted key physicochemical properties. Previous reports have determined that dynamic flow is capable of modifying the protein corona $[29,30]$, which could disrupt both AgNP agglomerate size and surface charge. As seen in Table 1, the low level dynamic movement had a negligible impact on both the extent of agglomeration and zeta potential measurements.

\subsection{Dynamic Flow-Modified AgNP Cellular Interactions}

Next, the influence of dynamic flow on AgNP deposition was investigated, for both the adherent A549 and the suspension U937 cell models. As seen in Figure 2, dynamic flow significantly altered NP association efficiencies, though in opposite modalities for these culture types. For A549 cells, the presence of lateral fluid flow decreased AgNP deposition by approximately $33 \%$. The $50 \mathrm{~nm}$ AgNPs would have been associated with a significant rate of sedimentation, thereby producing a high number of AgNP-A549 interactions [35]. Under dynamic flow, the lateral convective movement kept the AgNPs in suspension, resulting in the observed deposition decrease. For the suspension U937 cells, the fluid dynamics, which counterbalanced downward sedimentation effects, resulted in augmented AgNP cellular association. As shown in Figure 2, when U937 underwent AgNP exposure under dynamic conditions, there was an approximate $66 \%$ increase in NP cellular binding. 


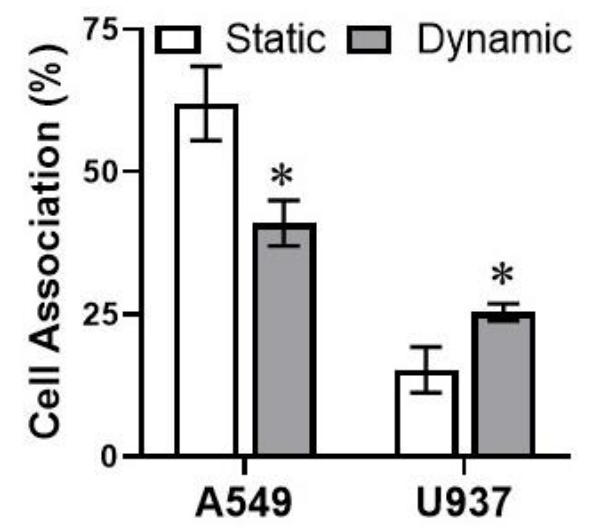

Figure 2. AgNP cellular association varies as a function of flow environment and cell model. Following $24 \mathrm{~h}$ exposure, AgNP interactions with the adherent A549 or suspension U937 models were measured within both static and dynamic environments. * indicates significance between static and dynamic conditions. $n=3, p<0.05$.

\subsection{AgNP-Induced Cytotoxicity Varied with Flow Condition}

As AgNPs are known to induce mammalian cytotoxicity [8-11], the first endpoint assessed was cellular viability, as a function of flow condition (Figure 3). Starting with the A549s (Figure 3A), the lower AgNP dosage produced a slight cytotoxic response. However, following $25 \mu \mathrm{g} / \mathrm{mL}$ exposure, there was an approximate $40 \%$ loss of cell viability under static conditions. Within a dynamic A549 model, the cells still displayed AgNP dosedependent cytotoxicity, though to a lesser degree, indicating that cellular damage was mitigated within a dynamic environment.
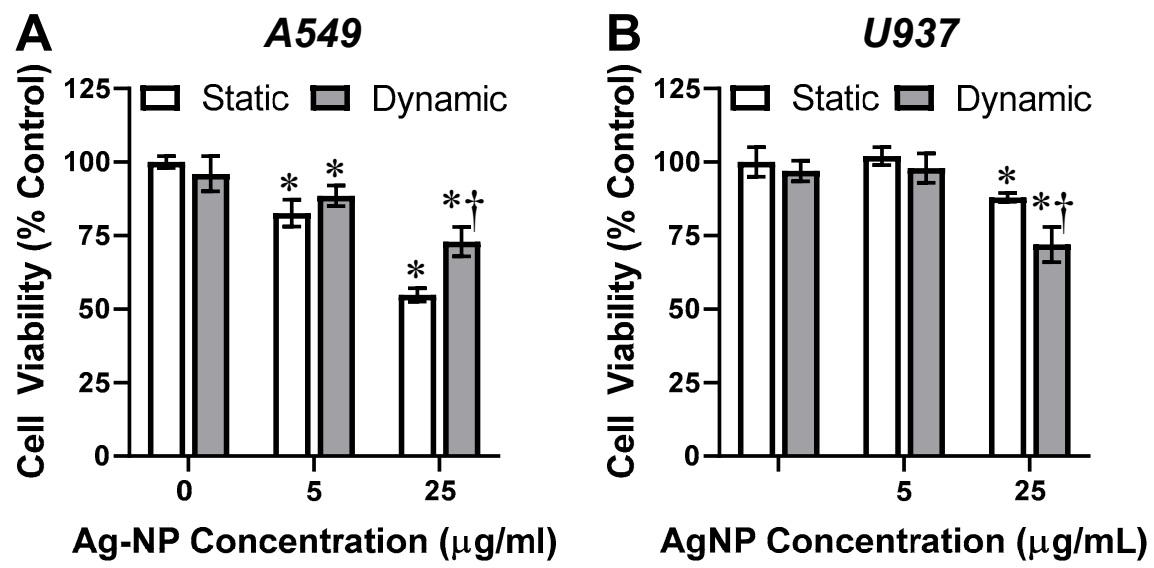

Figure 3. Cellular viability following AgNP exposure. Following $24 \mathrm{~h}$ AgNP exposure within either (A) A549 or (B) U937 models the cellular viability was assessed. These results indicated that within both systems viability was a function of AgNP dosage and flow condition. * and + indicate statistical significance compared to the untreated control and between static and dynamic conditions, respectively. $n=3, p<0.05$.

The U937 cells also demonstrated a dose-dependent cytotoxicity response following AgNP exposure, as expected. However, when examining the U937 dynamic flow results, opposite trends were identified (Figure 3B). Following $25 \mu \mathrm{g} / \mathrm{mL}$ exposure, the U937s experienced a mild $12 \%$ toxicity under static conditions. With the inclusion of dynamic flow, this toxicity rate increased to approximately $25 \%$, presumably due to amplified AgNPU937 interactions. These viability results are in excellent agreement with the AgNP cellular association profiles, which for A549 and U937 cells identified a respective decrease and 
increase in nano-cellular associations. Moreover, any cellular changes to cytotoxicity were not directly related to the presence of fluid dynamics as shown in controls.

\subsection{Intracellular Oxidative Stress Levels}

As the presence of dynamic flow was able to modulate AgNP-dependent cytotoxicity, the next goal was to explore if this phenomena translated to intracellular responses, which precede cell death. Based on the dose-dependent toxicity analysis, an exposure of $5 \mu \mathrm{g} / \mathrm{mL}$ AgNP was selected, as it did not elicit a strong degree of cellular death. Detection of ROS was used to monitor intracellular oxidative stress levels, as it is a known cellular response following NP exposure and an early indicator of apoptosis [40,41]. In the absence of AgNPs, ROS production was equivalent for static and dynamic conditions for both models (Figure 4). These controls demonstrated that dynamic flow did not impact basal A549 or U937 stress levels.
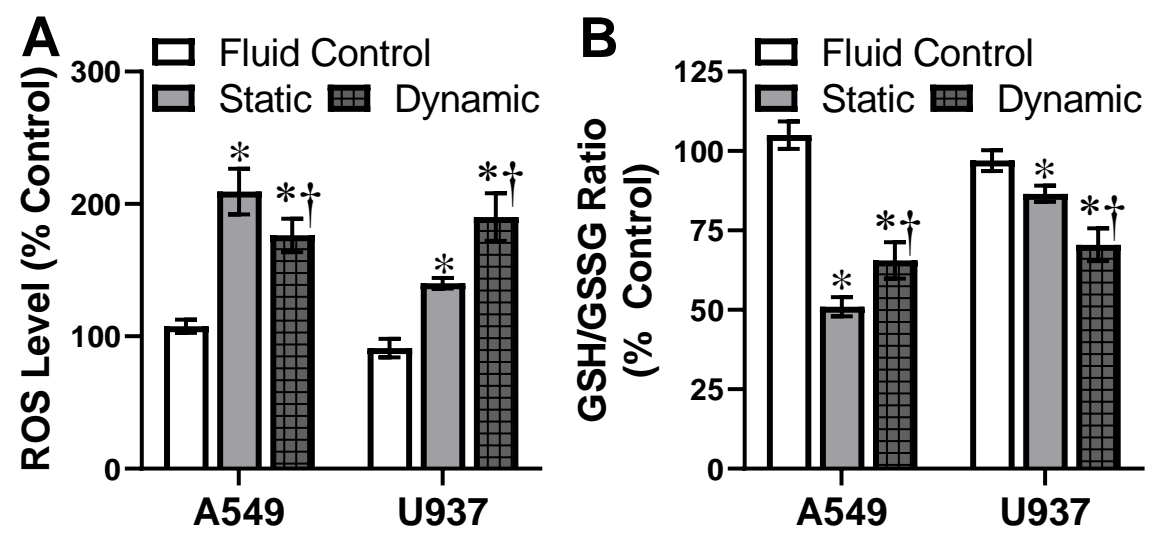

Figure 4. AgNP-induced intracellular oxidative stress levels. Both (A) ROS and (B) GSH/GSSG levels were used to monitor A549 and U937 intracellular oxidative stress following $5 \mu \mathrm{g} / \mathrm{mL}$ AgNP exposure for $24 \mathrm{~h}$. Experimentation was carried out under both static and dynamic conditions. Dynamic flow without AgNP conditions were also included as fluid control. Oxidative stress levels were found to be a function of cell type and flow condition. * and + indicate statistical significance compared to the untreated control and between static and dynamic conditions, respectively. $n=3$, $p<0.05$.

As shown in Figure 4A, $50 \mathrm{~nm}$ AgNP exposure within a static A549 model demonstrated elevated ROS levels. In agreement with the cell viability data, under dynamic conditions the A549 stress response was significantly decreased. Following static AgNP exposure, U937 cells demonstrated a mild ROS response, indicating active intracellular oxidative stress. However, when U937 cells underwent the same AgNP dosage exposure under dynamic conditions, the resultant ROS levels were augmented by approximately $50 \%$.

Intracellular glutathione exists in two states, reduced (GSH) and oxidized (GSSG). In normal, healthy cells, over $90 \%$ of all glutathione is in the GSH state. When cells are experiencing stress, the GSSG level rises; therefore, a decreased GSH/GSSG ratio is indicative of augmented oxidative stress [42,43]. As seen in Figure 4B, both A549 and U937 were experiencing stress following AgNP exposure under static conditions, due to GSH/GSSG ratios that were lower than the untreated control. Upon the introduction of fluid dynamics, GSH/GSSG ratios increased and decreased in the A549 and U937 cells systems, respectively, in agreement with the ROS data.

\subsection{Activation of Oxidative Stress Protein Markers}

Following identification of differential activation of ROS and glutathione responses within a dynamic environment, the next assessments targeted key proteins involved in the intracellular stress pathways. The goal was to elucidate whether $\mathrm{p} 53$ and $\mathrm{NF} \kappa \mathrm{B}$ were activated following AgNP exposure and whether the presence of dynamic flow modified 
the degree of this stress response. The targets of p53 and NFKB were specifically selected as these proteins have been previously shown to be induced following AgNP exposure in vitro $[44,45]$. As seen in Figure 5A, p53 was activated in both A549 and U937 systems following AgNP exposure. The phosphorylation of p53 mirror the ROS and glutathione results, with dynamic flow decreasing and increasing stress levels within the adherent and suspension models, respectively. When looking at NFkB activation, A549 showed both the AgNP-dependent stimulation and flow-dependent effects. However, U937 cells did not show any change in NFKB expression or activation following AgNP introduction, indicating that this pathway was not influenced by NP presence. Taken together, these results confirmed that exposure environment does influence intracellular oxidative stress proteins and pathways, though through cell-specific mechanisms.
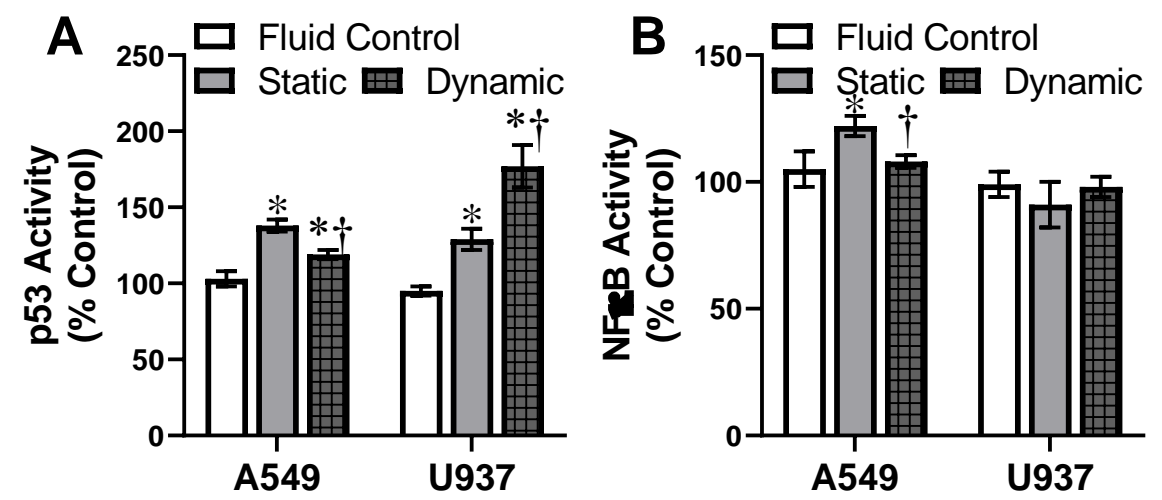

Figure 5. Activation of oxidative stress protein markers. Following exposure to $5 \mu \mathrm{g} / \mathrm{mL} \mathrm{AgNP}$, under both static and dynamic conditions, the A549 and U937 cells underwent evaluation for (A) p53 and (B) NFKB activation. Activation was determined by quantifying the phosphorylated state of target intracellular proteins, normalized by the total amounts of that protein. Activation of chosen oxidative stress pathways varied with cell type and exposure environment. Dynamic flow without AgNP conditions was also included as fluid control. * and + indicate statistical significance compared to the untreated control and between static and dynamic conditions, respectively. $n=3, p<0.05$.

\subsection{Secretion of Pro-Inflammatory Cytokines Following AgNP Exposure In Vitro}

AgNP-dependent acute stress induction has been linked to the activation of inflammatory responses in mammalian cells [46]. Once activated, an early inflammatory response is the production and secretion of pro-inflammatory cytokines, including IL-1 $\beta$ and TNF$\alpha$ [47]. For untreated controls, the IL-1 $\beta$ and TNF- $\alpha$ levels were equivalent for static and dynamic conditions, indicating that fluid flow did not alter cytokine production (Figure 6). As A549 cells are alveolar epithelial, they only secrete a basal, low-level amount of cytokines. As shown in Figure 6, AgNPs did not elicit a significant inflammatory response under any condition. However, U937 monocytes, which are a part of the immune system, produced a robust array of cytokines following activation, including IL-1 $\beta$ and TNF- $\alpha$. Regardless of exposure environment, $5 \mu \mathrm{g} / \mathrm{mL}$ AgNPs initiated a pro-inflammatory response, through upregulated cytokine production. Under dynamic conditions, a small increase in production levels was identified, indicating some degree of behavioral variance resulting from fluid flow conditions. 

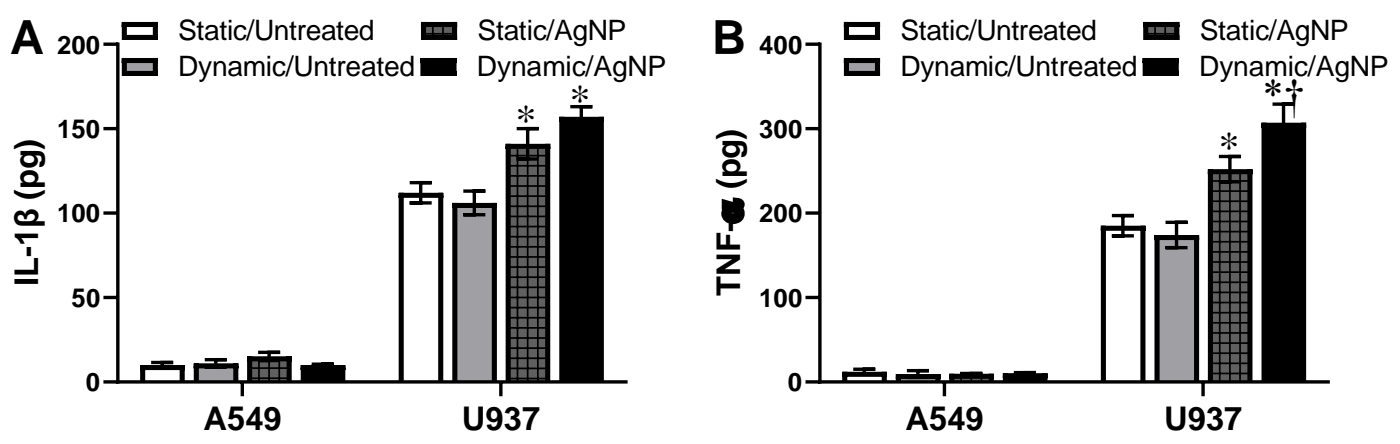

Figure 6. Secretion of pro-inflammatory cytokines following AgNP stimulation. The alveolar epithelial A549 and monocytic U937 cell lines were exposed to the denoted combinations of static/dynamic conditions and $5 \mu \mathrm{g} / \mathrm{mL}$ AgNPs. After $24 \mathrm{~h}$ the media were recovered and evaluated for levels of the pro-inflammatory cytokines of (A) IL-1 $\beta$ and (B) TNF- $\alpha .{ }^{*}$ and $\dagger$ indicate statistical significance compared to the untreated control and between static and dynamic conditions, respectively. $n=3, p<0.05$.

\section{Discussion}

The goal of this work was to elucidate the roles of cellular type (adhesion vs. suspension) and fluid dynamics in the AgNP cellular interactions and subsequent bioresponses, with a focus on oxidative stress markers. The results presented in this study demonstrated that both cellular and exposure characteristics significantly impacted the nano-cellular interface and the biological response. Therefore, each of these influences is discussed in greater detail below. Moreover, how this work supports the need and highlights implications for more physiologically relevant NP exposure models are included as points of discussion.

\subsection{Influence of Fluid Dynamics on Different Cell Classifications}

The addition of forced convective flow increased the biological relevance of the in vitro system, as all animal models are dynamic due to the presence of cardiovascular systems. By recreating a capillary-based velocity within the tubing, this model established diffusionlevel transport across the A549 surface or alongside the U937s, representing tissue-level shear rates experienced in vivo [48]. This study determined that the addition of dynamic flow altered the NP transport profiles, as the lateral force counteracts the downward sedimentation and random diffusion which dominate under static conditions [49]. Firstly, when examining the impact of fluid flow, it was found that the utilized volumetric flowrate did not alter AgNP physicochemical properties (Table 1). These results suggest that all the elucidated cellular modifications were due to an altered nano-cellular interface and not the AgNPs themselves.

For both the adherent A549 and the suspension U937 cells models, the addition of dynamic flow was found to impact nano-cellular interactions, AgNP-dependent cytotoxicity, and subsequent oxidative stress responses. However, inclusion of dynamic flow in the exposure environment had opposite impacts on the A549 and U937 models, due to their differential growth behaviors. For the adherent A549 cells, dynamic flow reduced AgNP deposition, whereas nano-cellular associations were increased for the U937 model. In agreement with previous studies from the literature, the level of AgNP interaction with the surrounding biological environment dictated the degree of cytotoxicity and oxidative stress [50,51]. As such, when AgNP exposure occurred within a dynamic A549 model, the cytotoxicity and array of oxidative stress markers were assuaged. On the contrary, the higher rate of AgNP and U937 collisions when both were flowing in the dynamic model resulted in augmented toxicity and stress pathways.

Looking beyond toxicity, this study focused on numerous oxidative stress markers, including ROS, glutathione levels, p53, NFkB, and cytokine secretion. Taken together, these pathways highlight a multitude of differential intracellular stress responses. These endpoints were found to vary as a function of both exposure environment and cell model, 
mirroring the cytotoxicity results. This study is the first to our knowledge that has explored the role of fluid dynamics on the nano-cellular interface and oxidative stress within a suspension-based in vitro model, highlighting the variance in cellular behavior. Of particular importance is the ability of AgNPs and the exposure environment to influence the secretion of pro-inflammatory cytokines from U937 cells, which introduce the potential for a systemic inflammatory response and long-term health consequences [52].

\subsection{The Need for Improved In Vitro Models}

The results presented in this study highlight the necessity of developing in vitro models that are more biologically relevant and are able to better assess how NPs will behave within in vivo environments. The need for enhanced systems is further supported by the conflicting results and poor correlation between cell- and animal-based models with regards to nanotoxicity and the induction of intracellular stress and signaling pathways [20-24]. Discrepancies between these models are due in part to the fact that NP physicochemical properties and dosimetry are dependent upon environmental factors. For example, when NPs are dispersed in physiological fluids, such as interstitial and lysosomal, the rate of ionic dissolution and extent of agglomeration are significantly altered versus cell culture media $[53,54]$. Additionally, NP exposure within a three-dimensional in vitro model diminished the extent of cytotoxic responses versus two-dimensional models, owing to the need for NPs to translocate through the cell systems prior to internalization [55]. In agreement with this work, fluid flow has previously been found to modify cellular morphology, dosimetry, and resultant bioresponses [25-27].

In addition to further validating the need for more physiologically representative exposure scenarios, this work determined that the impact of fluid dynamics on NP dosimetry and dependent oxidative stress varied with cell system behavior. The results that emerged from this dynamic model better align with published in vivo data, in which AgNP exposure at lower dosages elicits only a minor stress response, without a large induction of cytotoxicity [56,57]. Therefore, this work indicates that the addition of dynamic flow increases the relevance of in vitro models and has the potential to improve predictive modeling capabilities.

\section{Conclusions}

This study explored the role of cell adhesion properties and dynamic fluid movement on NP cellular interactions, cytotoxicity, and intracellular oxidative stress pathways following exposure to AgNPs. This work revealed that fluid flow modified the nano-bio interface but through differential mechanisms for adherent and suspension cells. For adherent cells, dynamic flow reduced AgNP contact and subsequent bioresponses due to a disruption of sedimentation. In contrast, cells that grow in suspension were associated with higher AgNP contact and oxidative stress activation within a dynamic exposure environment. The model implemented in this work, which incorporated dynamic flow, demonstrates how enhanced in vitro systems capture physiological influences and can thereby produce differential biological responses. Alterations to in vitro models also allow experimental flexibility and the ability to tailor design characteristics. For example, this study focused on low, diffusion-based flow that is associated with diffusion-level circulation. However, by switching to an endothelial cell model and increasing fluid flow rates, an arterial exposure system could be recreated.

Supplementary Materials: The following are available online at https:/ / www.mdpi.com/article / 10.3390/antiox10060832/s1, Figure S1: Cytotoxicity Dynamic Controls; Figure S2: Oxidative Stress Dynamic Controls; Figure S3: Cytokine Secretion Dynamic Controls.

Author Contributions: Conceptualization, K.E.B. and K.A.K.; methodology, K.E.B., R.F.U., and K.A.K.; investigation, K.E.B., R.F.U., M.E.J., and M.F.B.; formal analysis, K.E.B. and K.A.K.; writingoriginal draft preparation, K.E.B. and M.E.J.; writing-review and editing, K.A.K.; visualization, 
K.E.B. and K.A.K.; supervision, K.A.K.; project administration, K.A.K.; funding acquisition, K.E.B. and K.A.K. All authors have read and agreed to the published version of the manuscript.

Funding: K.E.B., K.A.K., and this work were funded in part by the Dayton Area Graduate Studies Institute, award RH-16-6. R.F.U. was funded by the University of Dayton Honors Program. M.E.J. was funded by the Claire Boothe Luce Foundation.

Institutional Review Board Statement: Not applicable.

Informed Consent Statement: Not applicable.

Data Availability Statement: All data are contained in this article and supplementary materials.

Conflicts of Interest: The authors declare no conflict of interest. The funders had no role in the design of the study; in the collection, analyses, or interpretation of data; in the writing of the manuscript, or in the decision to publish the results.

\section{References}

1. Kessler, R. Engineered nanoparticles in consumer products: Understanding a new ingredient. Environ. Health Perspect. 2011, 119, A120-A125. [CrossRef] [PubMed]

2. Yaqoob, A.A.; Umar, K. Silver nanoparticles: Various methods of synthesis, size affecting factors and their potential applications-A review. Appl. Nanosci. 2020, 10, 1369-1378. [CrossRef]

3. Zhang, X.F.; Liu, Z.G.; Shen, W.; Gurunathan, S. Silver nanoparticles: Synthesis, characterization, properties, applications, and therapeutic approaches. Int. J. Mol. Sci. 2016, 17, 1534.

4. Das, C.A.; Kumar, V.G.; Dhas, T.S.; Karthick, V.; Govindaraju, K.; Joselin, J.M.; Baalamurugan, J. Antibacterial activity of silver nanoparticles (biosynthesis): A short review on recent advances. Biocatal. Agric. Biotechnol. 2020, 27, 101593.

5. Ge, L.; Li, Q.; Wang, M.; Ouyang, J.; Li, X.; Xing, M.M.Q. Nanosilver particles in medical applications: Synthesis, performance, and toxicity. Int. J. Nanomed. 2014, 9, 2399-2407.

6. Momeni, M.M.; Shayeb, Y.; Gheibee, S. Silver nanoparticles decorated titanium dioxide-tungsten trioxide nanotube films with enhanced visible light photo catalytic activity. Ceram. Int. 2017, 43, 564-570.

7. Akter, M.; Sikder, M.T.; Rahman, M.M.; Ullah, A.A.; Hossain, K.F.B.; Banik, S.; Hosokawa, T.; Saito, T.; Kurasaki, M. A systematic review on silver nanoparticles-induced cytotoxicity: Physicochemical properties and perspectives. J. Adv. Res. 2018, 9, 1-16.

8. Breitner, E.K.; Burns, K.E.; Hussain, S.M.; Comfort, K.K. Implementation of physiological fluids to provide insight into the characterization, fate, and biological interactions of silver nanoparticles. Nanotechnology 2018, 29, 254001. [CrossRef] [PubMed]

9. Comfort, K.K.; Braydich-Stolle, L.K.; Maurer, E.I.; Hussain, S.M. Less is more: Long-term in vitro exposure to low levels of silver nanoparticles provides new insights for nanomaterial evaluation. ACS Nano 2014, 8, 3260-3271.

10. Comfort, K.K.; Maurer, E.I.; Hussain, S.M. Slow release of ions from internalized silver nanoparticles modifies the epidermal growth factor signaling response. Colloids Surf. B Biointerfaces 2014, 123, 136-142. [CrossRef] [PubMed]

11. Hadrup, N.; Lam, H.R. Oral toxicity of silver ions, silver nanoparticles and colloidal silver-A review. Regul. Toxicol. Pharm. 2014, 68, 1-7. [CrossRef] [PubMed]

12. Khan, A.M.; Korzeniowska, B.; Gorshkov, V.; Tahir, M.; Schrøder, H.; Skytte, L.; Rasmussen, K.L.; Khandige, S.; Møller-Jensen, J.; Kjeldsen, F. Silver nanoparticle-induced expression of proteins related to oxidative stress and neurodegeneration in an in vitro human blood-brain barrier model. Nanotoxicology 2019, 13, 221-239. [CrossRef] [PubMed]

13. León-Silva, S.; Fernández-Luqueño, F.; López-Valdez, F. Silver nanoparticles (AgNP) in the environment: A review of potential risks on human and environmental health. Water Air Soil Pollut. 2016, 227, 1-20. [CrossRef]

14. Li, J.; Tang, M.; Xue, Y. Review of the effects of silver nanoparticle exposure on gut bacteria. J. Appl. Toxicol. 2019, $39,27-37$.

15. Modena, M.M.; Rühle, B.; Burg, T.P.; Wuttke, S. Nanoparticle characterization: What to measure. Adv. Mater. 2019, 31, 1901556. [CrossRef]

16. Hussain, S.M.; Warheit, D.B.; Ng, S.P.; Comfort, K.K.; Grabinski, C.M.; Braydich-Stolle, L.K. At the crossroads of nanotoxicology in vitro: Past achievements and current challenges. Toxicol. Sci. 2015, 147, 5-16. [CrossRef]

17. Enea, M.; Pereira, E.; Costa, J.; Soares, M.E.; da Silva, D.D.; de Lourdes Bastos, M.; Carmo, H.F. Cellular uptake and toxicity of gold nanoparticles on two distinct hepatic cell models. Toxicol. In Vitro 2021, 70, 105046. [CrossRef]

18. Soenen, S.J.; De Cuyper, M. Assessing iron oxide nanoparticle toxicity in vitro: Current status and future prospects. Nanomedicine 2010, 5, 1261-1275. [CrossRef]

19. Clift, M.J.D.; Gehr, P.; Rothen-Rutishauser, B.R. Nanotoxicology: A perspective and discussion of whether or not in vitro testing is a valid alternative. Arch. Toxicol. 2011, 85, 723-731. [CrossRef]

20. Demokritou, P.; Gass, S.; Pyrgiotakis, G.; Cohen, J.M.; Goldsmith, W.; McKinney, W.; Frazer, D.; Ma, J.; Schwegler-Berry, D.; Brain, $\mathrm{J} . ;$ et al. An in vivo and in vitro toxicological characterization of realistic nanoscale $\mathrm{CeO}_{2}$ inhalation exposures. Nanotoxicology 2013, 7, 1338-1350. [CrossRef] [PubMed]

21. Frohlich, E.; Salar-Behzadi, S. Toxicological assessment of inhaled nanoparticles: Role of in vivo, ex vivo, in vitro, and in silico studies. Int. J. Mol. Sci. 2014, 15, 4795-4822. [CrossRef] [PubMed] 
22. Jia, Y.P.; Ma, B.Y.; Wei, X.W.; Qian, Z.Y. The in vitro and in vivo toxicity of gold nanoparticles. Chin. Chem. Lett. 2017, 28, 691-702. [CrossRef]

23. Hulsart-Billström, G.; Dawson, J.I.; Hofmann, S.; Müller, R.; Stoddart, M.J.; Alini, M.; Redl, H.; El Haj, A.; Brown, R.; Salih, V.; et al. A surprisingly poor correlation between in vitro and in vivo testing of biomaterials for bone regeneration: Results of a multicentre analysis. Eur. Cell Mater. 2016, 31, 312-322. [CrossRef] [PubMed]

24. Kumar, V.; Sharma, N.; Maitra, S.S. In vitro and in vivo toxicity assessment of nanoparticles. Int. Nano Lett. 2017, 7, 243-256. [CrossRef]

25. Breitner, E.K.; Hussain, S.M.; Comfort, K.K. The role of biological fluid and dynamic flow in the behavior and cellular interactions of gold nanoparticles. J. Nanobiotechnol. 2015, 13, 56. [CrossRef] [PubMed]

26. Fede, C.; Fortunati, I.; Weber, V.; Rossetto, N.; Bertasi, F.; Petrelli, L.; Guidolin, D.; Signorini, R.; De Caro, R.; Albertin, G.; et al. Evaluation of gold nanoparticles toxicity toward human endothelial cells under static and flow conditions. Microvas Res. 2015, 97, 147-155. [CrossRef]

27. Ucciferri, N.; Collnot, E.M.; Gaiser, B.K.; Tirella, A.; Stone, V.; Domenici, C.; Lehr, C.M.; Ahluwalia, A. In vitro toxicology screening of nanoparticles on primary human endothelial cells and the role of flow in modulating cell response. Nanotoxicology 2014, 8 , 697-708. [CrossRef]

28. Hajal, C.; Campisi, M.; Mattu, C.; Chiono, V.; Kamm, R.D. In vitro models of molecular and nano-particle transport across the blood-brain barrier. Biomicrofluidics 2018, 12, 042213. [CrossRef]

29. Braun, N.J.; DeBrosse, M.C.; Hussain, S.M.; Comfort, K.K. Modification of the protein corona-nanoparticle complex by physiological factors. Mater. Sci. Eng. C 2016, 64, 34-42. [CrossRef]

30. Abbina, S.; Takeuchi, L.E.; Anilkumar, P.; Yu, K.; Rogalski, J.C.; Shenoi, R.A.; Constantinescu, I.; Kizhakkedathu, J.N. Blood circulation of soft nanomaterials is governed by dynamic remodeling of protein opsonins at nano-biointerface. Nat. Commun. 2020, 11, 1-12. [CrossRef]

31. Brandão, F.; Fernández-Bertólez, N.; Rosário, F.; Bessa, M.J.; Fraga, S.; Pásaro, E.; Teixeira, J.P.; Laffon, B.; Valdiglesias, V.; Costa, C. Genotoxicity of tio2 nanoparticles in four different human cell lines (A549, HEPG2, A172 and SH-SY5Y). Nanomaterials 2020, 10, 412. [CrossRef] [PubMed]

32. Sahu, D.; Kannan, G.M.; Vijayaraghavan, R. Size-dependent effect of zinc oxide on toxicity and inflammatory potential of human monocytes. J. Toxicol. Environ. Health Part A 2014, 77, 177-191. [CrossRef]

33. Berger, S.A.; Goldsmith, W.; Lewis, E.R. Introduction to Bioengineering; Oxford University Press: Oxford, UK, 1996.

34. Zook, J.M.; Long, S.E.; Cleveland, D.; Geronimo, C.L.; MacCuspie, R.I. Measuring silver nanoparticle dissolution in complex biological and environmental matrices using UV-visible absorbance. Anal. Bioanal. Chem. 2011, 401, 1993-2002. [CrossRef] [PubMed]

35. Schulz, J.B.; Lindenau, J.; Seyfried, J.; Dichgans, J. Glutathione, oxidative stress and neurodegeneration. Eur. J. Biochem. 2000, 267, 4904-4911. [CrossRef] [PubMed]

36. Cho, E.J.; Holback, H.; Liu, K.C.; Abouelmagd, S.A.; Park, J.; Yeo, Y. Nanoparticle characterization: State of the art, challenges, and emerging technologies. Mol. Pharm. 2013, 10, 2093-2110. [CrossRef]

37. Kim, W.; Kim, W.K.; Lee, K.; Son, M.J.; Kwak, M.; Chang, W.S.; Min, J.K.; Song, N.W.; Lee, J.; Bae, K.H. A reliable approach for assessing size-dependent effects of silica nanoparticles on cellular internalization behavior and cytotoxic mechanisms. Int. J. Nanomed. 2019, 14, 7375. [CrossRef]

38. Luby, A.O.; Breitner, E.K.; Comfort, K.K. Preliminary protein corona formation stabilizes gold nanoparticles and improves deposition efficiency. Appl. Nanosci. 2016, 6, 827-836. [CrossRef]

39. Munir, T.; Mahmood, A.; Imran, M.; Sohail, A.; Fakhar-e-Alam, M.; Sharif, M.; Masood, T.; Bajwa, S.Z.; Shafiq, F.; Latif, S. Quantitative analysis of glucose by using (PVP and MA) capped silver nanoparticles for biosensing applications. Phys. B Condens. Matter 2021, 602, 412564. [CrossRef]

40. Labrador-Rached, C.J.; Browning, R.T.; Braydich-Stolle, L.K.; Comfort, K.K. Toxicological implications of platinum nanoparticle exposure: Stimulation of intracellular stress, inflammatory response, and akt signaling in vitro. J. Toxicol. 2018, 2018, 1367801. [CrossRef] [PubMed]

41. Redza-Dutordoir, M.; Averill-Bates, D.A. Activation of apoptosis signaling pathways by reactive oxygen species. Biochem. Biophys. Acta Mol. Cell Res. 2016, 1863, 2977-2992. [CrossRef]

42. Dasgupta, N.; Ranjan, S.; Mishra, D.; Ramalingam, C. Thermal co-reduction engineered silver nanoparticles induce oxidative cell damage in human colon cancer cells through inhibition of reduced glutathione and induction of mitochondria-involved apoptosis. Chemico-Biol. Interact. 2018, 295, 109-118. [CrossRef] [PubMed]

43. Matschke, V.; Theiss, C.; Matschke, J. Oxidative stress: The lowest common denominator of multiple diseases. Neural Regen. Res. 2019, 14, 238-241. [CrossRef] [PubMed]

44. Blanco, J.; Tomás-Hernández, S.; García, T.; Mulero, M.; Gómez, M.; Domingo, J.L.; Sánchez, D.J. Oral exposure to silver nanoparticles increases oxidative stress markers in the liver of male rats and deregulates the insulin signalling pathway and p53 and cleaved caspase 3 protein expression. Food Chem. Toxicol. 2018, 115, 398-404. [CrossRef] [PubMed]

45. Fehaid, A.; Fujii, R.; Sato, T.; Taniguchi, A. Silver nanoparticles affect the inflammatory response in a lung epithelial cell line. Open Biotechnol. J. 2020, 14, 113-123. [CrossRef] 
46. Barbasz, A.; Oćwieja, M.; Roman, M. Toxicity of silver nanoparticles towards tumoral human cell lines U-937 and HL-60. Coll. Surf. B Biointerfaces 2017, 156, 397-404. [CrossRef]

47. Kongseng, S.; Yoovathaworn, K.; Wongprasert, K.; Chunhabundit, R.; Sukwong, P.; Pissuwan, D. Cytotoxic and inflammatory responses of TiO2 nanoparticles on human peripheral blood mononuclear cells. J. Appl. Toxicol. 2016, 36, 1364-1373. [CrossRef]

48. Roignot, J.; Peng, X.; Mostov, K. Polarity in mammalian epithelial morphogenesis. Cold Spring Harb. Perspect. Biol. 2013, 5 , a013789. [CrossRef]

49. van Silfhout, A.; Erné, B. Magnetic detection of nanoparticle sedimentation in magnetized ferrofluids. J. Magn. Magn. Mater. 2019, 472, 53-58. [CrossRef]

50. De Matteis, V.; Rinaldi, R. Toxicity assessment in the nanoparticle era. Adv. Exp. Med. Biol. 2018, 1048, 1-19.

51. Savage, D.T.; Hilt, J.Z.; Dziubla, T.D. In Vitro methods for assessing nanoparticle toxicity. Methods Mol. Biol. $2019,1894,1-29$.

52. Kany, S.; Vollrath, J.T.; Relja, B. Cytokines in inflammatory disease. Int. J. Mol. Sci. 2019, 20, 6008. [CrossRef]

53. Cathe, D.S.; Whitaker, J.N.; Breitner, E.K.; Comfort, K.K. Exposure to metal oxide nanoparticles in physiological fluid induced synergistic biological effects in a keratinocyte model. Toxicol. Lett. 2017, 268, 1-7. [CrossRef] [PubMed]

54. Cho, W.S.; Duffin, R.; Howie, S.E.M.; Scotton, C.J.; Wallace, W.A.H.; MacNee, W.; Bradley, M.; Megson, I.L.; Donaldson, K. Progressive severe lung injury by zinc oxide nanoparticles; the role of Zn2+ dissolution inside lysosomes. Part. Fibre Toxicol. 2011, 8, 27. [CrossRef] [PubMed]

55. Hoelting, L.; Scheinhardt, B.; Bondarenko, O.; Schildknecht, S.; Kapitza, M.; Tanavde, V.; Tan, B.; Lee, Q.Y.; Mecking, S.; Leist, M.; et al. A 3-dimensional embryonic stem cell (hESC)-derived model to detect developmental neurotoxicity of nanoparticles. Arch. Toxicol. 2015, 87, 721-733. [CrossRef]

56. Park, E.J.; Bae, E.; Yi, J.; Kim, Y.; Choi, K.; Lee, S.H.; Yoon, J.; Lee, B.C.; Park, K. Repeated-dose toxicity and inflammatory responses in mice by oral administration of silver nanoparticles. Environ. Toxicol. Pharmacol. 2010, 30, 162-168. [CrossRef]

57. van den Brule, S.; Ambroise, J.; Lecloux, H.; Levard, C.; Soulas, R.; de Temmerman, P.J.; Palmai-Pallag, M.; Marbaix, E.; Lison, D. Dietary silver nanoparticles can disturb the gut microbiota in mice. Part. Fibre Toxicol. 2016, 13, 38. [CrossRef] [PubMed] 